\title{
Novel Integrated Optical Displacement Sensor For Scanning Force Microscopies
}

\author{
Atilla Aydinli, Isa Kiyat, and Coskun Kocabas \\ Department of Physics \\ Bilkent University \\ 06800, Ankara, Turkey \\ Email: aydinli@fen.bilkent.edu.tr
}

\begin{abstract}
A novel displacement sensor for scanning force microscopies using an integrated optical micro-ring resonator is deseribed. Device operates by monitoring the changes in transmission spectrum of micro-ring resonator. This design provides sensitivities about $\sim 10^{-4} \AA^{-1}$.
\end{abstract}

\section{INTRODUCTION}

In this article, we introduce integrated optical detection for scanning force microscopies. This method has many advantages over others. First, an integrated sensor does not require any alignment during scanning surface and it is possible to scan large areas. Second, integrated sensors are suitable for cantilever arrays due to their compactness, simplicity and potential for mass production. It should also be mentioned that integrated sensors such as piezo-resistive ones [1] have less sensitivity than the external sensors such as optical levers [2] . Using an integrated optical sensor, we expect to achieve as high sensitivity as external sensors. Integrated optical devices can be inexpensive and they can be used in harsh environments such as UHV systems and electromagnetically active environments. In this design, an optical waveguide coupled to a high finess micro-ring resonator integrated with a cantilever is used as a strain sensor to deduce displacement. Basically, stress due to displacement of the cantilever changes the local refractive index on the ring resonator through the photo-elastic effect, and index change cause modifications in the transmission characteristics of the optical waveguide coupled ring resonator. Monitoring the intensity modulation trough the optical waveguide, it is possible to determine the cantilever displacement with high accuracy. Fig.1 illustrates the the operational principle of the ring resonator displacement sensor.

\section{RING RESONATOR AS DISPLACEMENT SENSOR}

For the purpose of sensing displacement, we are mainly interested in modulation applications of ring resonators. It should be possible to obtain a large modulation in transmitted optical power by small variations of the refractive index. This kind of modulation is useful only if the resonance wavelength shift remains in the bandwidth of the resonator. Such shifts can only be achieved through a controllable change in optical path length of resonators, which is a function of resonator physical length and effective refractive index. Here, stress induced refractive index change is employed. When the ring

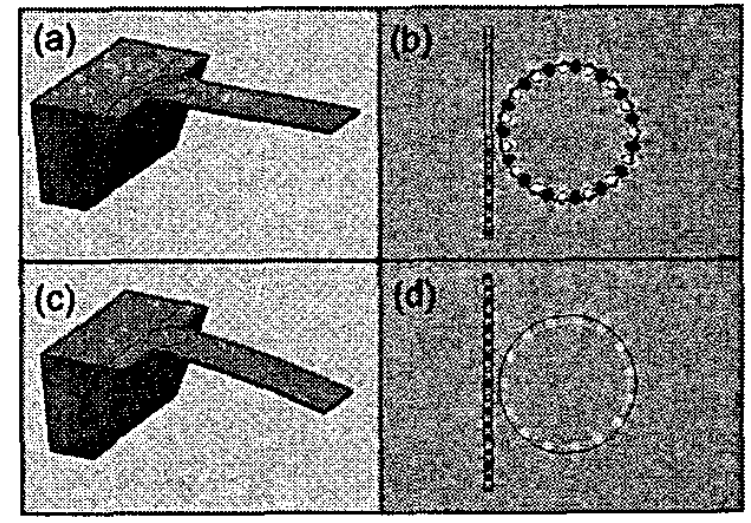

Fig. 1. A schematic illustration of the operational principle for the integrated micro-ring resonator displacement sensor, (a and c) shows the cantilever for unbend and bend condition, (b and d) shows the field distribution on the ring resonator on the cantilever.

resonator is designed to have a high- $Q$ factor, the modulation is dramatic due to steep fall of the transmission dip. A ring resonator could have high Q-factors when designed to work at the critical coupling regime.

As the operational principle of the ring resonator coupled waveguide sensor depends on the stress distribution along the cantilever,we have calculated the 3D stress distribution using Finite Element Method (FEM) simulations, for which; Young's Modulus of $0.83 \times 10^{11} \mathrm{~N} / \mathrm{m}^{2}$, and Poisson's ratio of 0.31 were used. Static analysis was performed using ANSYS software. The mesh was increased in the region where ring resonator was placed. The analysis performed here uses the stress at the surface. Maximum stress occurs at the supporting point of the cantilever and decreases linearly along the cantilever. In Fig. 2 we plot the stress distribution along the cantilever with an integrated ring resonator. Due to the photo-elastic effect, effective index changes due to the stress and through the equation,

$$
n_{e f f}=n_{0}+\sum_{i} C_{i} \sigma_{i}
$$

where, $C_{i}$ is the stress optic constant of waveguide and $\sigma_{i}$ is the local stress. From Fig.2b, it is clearly observed that stress 


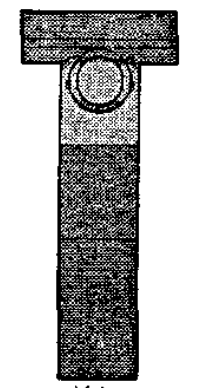

(a)

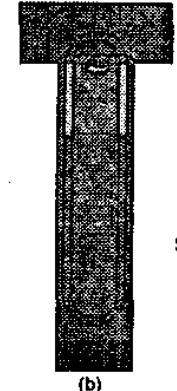

(b)

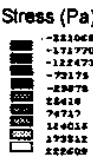

Fig. 2. Longitudinal stress distribution on the cantilevers with a ring (a) and a race-track (b) shape resonator. Long straight arms in the race-track resonators are useful for increasing the accumulated phase shift.

occurs on the two sides of the ring which are parallel with the major symmetry axis of the cantilever. In order to increase the accumulated phase shift, the parallel sides may be kept longer. The optimum design is a race-track shaped resonator, because of its two long parallel sides where stress is much larger than the curved sides. Fig.2b shows the stress contours on the race-track resonator. Phase shift can be approximated as;

$$
\Delta \Phi \simeq \frac{3 \pi C_{l} E t L_{r t}}{2 \lambda l^{2}} z
$$

where $L_{r t}$ is the length of the race-track resonator. From Eq. 2, it is observed that the phase shift is linearly proportional to the cantilever deflection and it is related to the cantilever geometry and mechanical properties of the cantilever material. For higher sensitivity, total length of the ring and the elastooptic coefficient of the material must be large. Phase shift can also be written as a function of force applied on the tip and,

$$
\Delta \Phi \simeq \frac{6 \pi C_{l} l L_{r t}}{\lambda w t^{2}} F
$$

where $F$ is the applied force, $w$ is the width of the cantilever. In order to increase the phase shift due to the applied force we have to keep the length long and the thickness of the cantilever small which results in a trade of between force and displacement sensitivity. It is not possible to achieve high displacement sensitivity as well as high force sensitivity. We can conclude that short and thick cantilever is sensitive to displacement measurements whereas long and thin cantilevers is more sensitive for force detection.

\section{Sensitivity ANALYSis}

The sensitivity of displacement sensors is the essential property of the atomic force microscopies. The minimum detectable force and the displacement depend on the sensitivity of the sensor and the noise of the system. Integrated sensors have less sensitivity than external ones. There have been many studies to increase the sensitivity of the integrated sensor, but the best sensitivity achieved so far is $\sim 10^{-6} \mathrm{~A}^{-1}$ [3] , which is two orders of magnitude less than the sensitivity of the interferometric detection [4]. Force sensitivity can be defined as the variation of the current on the detector per unit

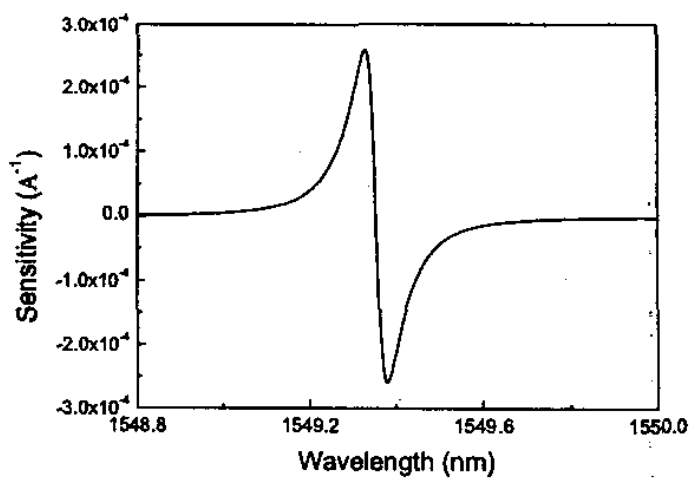

Fig. 3. Sensitivity vs wavelength for single bus ring resonator with critical coupling achieved.

force applied on the tip, with the same logic displacement sensitivity can be defined as the variation of the current on the detector per unit displacement of the cantilever. The detected power, $I_{t}$ depends on the transmission characteristics of the ring resonator coupled waveguide. We can write displacement and force sensitivities as

$$
\text { and } \begin{aligned}
S_{d} & =\beta \frac{\Delta I_{r}}{I_{0} \Delta z}=\beta \frac{1}{I_{0}} \frac{d I_{r}}{d \phi} \frac{d \phi}{d z} \\
S_{f} & =\beta \frac{\Delta I_{r}}{I_{0} \Delta F}=\beta \frac{1}{I_{0}} \frac{d I_{r}}{d \phi} \frac{d \phi}{d F}
\end{aligned}
$$

where $\beta$ is the correction factor. The highest sensitivities about $2.510^{-4} \AA^{-1}$ are achieved under critical coupling conditions. The calculated sensitivities are promising, and it is possible to achieve sensitivities as high as the sensitivity of the interferometric detection. Sensitivity of the detector is depends mainly on the design of the resonator and the waveguide. The displacement sensitivity also depends on the wavelength of the light source. Fig. 3 shows the sensitivity of the cantilever integrated with single bus ring resonator versus wavelength.

\section{CONCLUSION}

In this article, we propose a novel integrated ring resonator displacement sensor for scanning force microscopies. We design and analyze the feasibility of the integrated optical sensor. The concept based on elasto-optic effect is discussed. The design of the ring resonator was described and theoretical investigation of the force and displacement sensitivity was presented. We find that integrated optical sensor is attractive because of its high sensitivity about $10^{-4} \AA^{-1}$ and simplicity. We introduce a. new application area for integrated optics. This design is a good alternative for piezoresistive cantilevers especially in electromagnetically active environments. This method has high potential for improvement.

\section{REFERENCES}

[1] M. Tortonese et al,1991 Proceeding of IEEE conferance on Tranducers $91,448-451$

[2] G. Meyer, N. M. Amer, Appl. Phys. Lett. 53,(1988) 1045

[3] A. J. Brook, et al, J. Micromech. Microeng. 13,(2003), 124

[4] D. Rugar et al, Appl. Phys. Lett 55, (1989), 2588 